\title{
Study on Auto Disturbance Rejection Controller of HAPC Base on Parameters Optimization
}

\author{
Guo $\mathrm{Li}^{1, \mathrm{a}}$, Jianzhong Zhao ${ }^{2, \mathrm{~b}}$ \\ ${ }^{1}$ Automation School, University of Science and Technology in Beijing, Beijing, 100083, China \\ ${ }^{2}$ Automation School, University of Science and Technology in Beijing, Beijing, 100083, China \\ aemail:guo6396@sina.com, bemail:happyzjz1314@163.com
}

Keywords: HAPC; LADRC; Fuzzy Adaptive Algorithm; Monte-Carlo method

\begin{abstract}
This paper refers to the HAPC of 400mm single stand reversible cold rolling mill, establishing mathematical model, simplifying and reducing orders for system transfer function on the foundation of reasonable assumption. This paper also designs PID and LADRC for reducing order model and uses fuzzy adaptive to do online optimization for the parameters of LADRC. In the end, the paper shows the simulation program of the controllers mentioned above. The results show that LADRC has less overshoot, shorter settling time, better robustness and stronger ability to restrain the disturbance. The conclusion is that the control performance of LADRC is superior than that of PID.
\end{abstract}

\section{Introduction}

Hydraulic APC is one of the most important technologies in the modern rolling process, and it has an important position in the process of APC. Auto disturbance rejection control algorithm is a nonlinear control law proposed in recent years. It does not depend on the exact mathematical model of the controlled object, it can estimate the perturbation of the object model in real time, and using special nonlinear feedback structure for dynamic compensation.

However, the classical auto disturbance rejection controller has a large number of parameters to be adjusted, and the parameters tuning are mostly dependent on experience, which greatly hinders its application in practice. The linear auto disturbance rejection control algorithm is improved in linearity based on the classical auto disturbance rejection control algorithm. And it has the advantages of the classical auto disturbance rejection control, and simplifies the process of setting parameters. Therefore, it is a feasible and meaningful subject to study the control of hydraulic APC system based on the linear auto disturbance rejection control algorithm.

\section{Hydraulic APC System Model}

Hydraulic Automatic Position Control(HAPC) is a typical closed loop feedback control system, which is composed of displacement sensor to measure the displacement of hydraulic cylinder or the roll gap. HAPC is a high precision and high response electro-hydraulic position servo system. Its task is to accept the AGC system's instruction value, to carry out the position closed-loop control of the cylinder, so that makes the cylinder real-time and accurate positioning in the position required by the command to achieve the purpose of setting and controlling the no-load roll gap. The typical HAPC system consists of the controller, the servo amplifier, the electro-hydraulic servo valve and the displacement sensor, as shown in Fig.1.

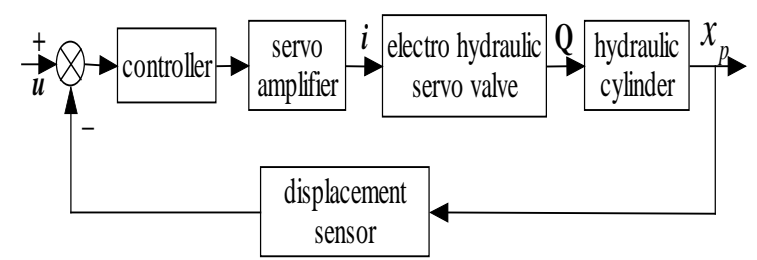

Fig.1. Control block diagram of HAPC system

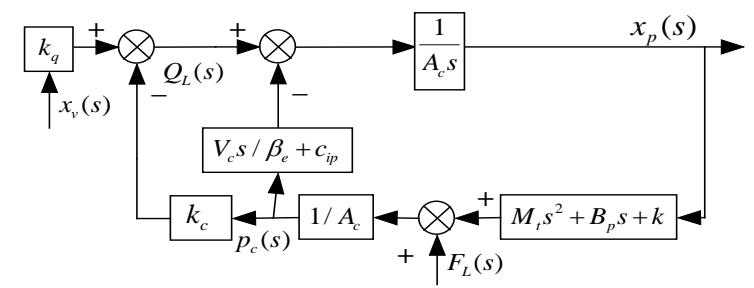

Fig.2. Valve controlled cylinder dynamic model 
Servo amplifier convert the input voltage signal into current signal to control the servo valve. When the current negative feedback servo amplifier is used, the current increment of $\Delta I$ is proportional to the input voltage increment $\Delta U . k_{a}=\frac{\Delta I}{\Delta U}$, where, $k_{a}$ is servo amplifier gain.

The bandwidth of electro hydraulic servo valve is similar to the natural frequency of hydraulic pressure, the transfer function of the electro hydraulic servo valve is regarded as two order oscillation. $G_{s v}=\frac{Q(s)}{I(s)}=\frac{k_{s v}}{s^{2} / \omega_{s v}^{2}+2 \xi_{s v} s / \omega_{s v}+1}$, where, $k_{s v}$ is servo valve flow gain, $\omega_{s v}$ is natural frequency of servo valve and $\xi_{s v}$ is damping ratio of servo valve.

The dynamic model of valve controlled asymmetrical hydraulic is shown as Fig.2. The comprehensive response equation of hydraulic cylinder output displacement $x_{p}$ about the valve displacement $x_{v}$ and any load force $F_{L}$ as shown as Eq1:

$$
x_{p}(s)=\frac{\frac{k_{q}}{A_{c}} x_{v}(s)-\frac{k_{c e}}{A_{c}^{2}}\left(1+\frac{V_{0}}{\beta_{e} k_{c e}} s\right) F_{L}(s)}{\frac{V_{0} M_{t}}{\beta_{e} A_{c}^{2}} s^{3}+\left(\frac{M_{t} k_{c e}}{A_{c}^{2}}+\frac{B_{p} V_{0}}{\beta_{e} A_{c}^{2}}\right) s^{2}+\left(1+\frac{B_{p} k_{c e}}{A_{c}^{2}}+\frac{k V_{0}}{\beta_{e} A_{c}^{2}}\right) s+\frac{k_{c e} k}{A_{c}^{2}}}
$$

Where, $k_{c e}=k_{c}+C_{i p}$. Because the damping coefficient $A_{c}^{2} / k$ is much greater than $B_{p}$, so the Eq1 can simplified as Eq2:

$$
x_{p}(s)=\frac{\frac{k_{q}}{A_{c}} x_{v}(s)-\frac{k_{c e}}{A_{c}^{2}}\left(1+\frac{V_{0}}{\beta_{e} k_{c e}} s\right) F_{L}(s)}{\frac{s^{3}}{\omega_{h}^{2}}+\frac{2 \xi_{h}}{\omega_{h}} s^{2}+\left(1+\frac{k}{k_{h}}\right) s+\frac{k_{c e} k}{A_{c}^{2}}}
$$

Where, $k_{h}$ is hydraulic spring rigidity, $k_{h}=\frac{\beta_{e} A_{c}^{2}}{V_{0}} \cdot \omega_{h}$ is hydraulic natural frequency, $\omega_{h}=\sqrt{\frac{\beta_{e} A_{c}^{2}}{V_{0} M_{t}}}$. $\xi_{h}$ is damping ratio, $\xi_{h}=\frac{k_{c e}}{2 A_{c}} \sqrt{\frac{\beta_{e} M_{t}}{V_{0}}}+\frac{B_{p}}{2 A_{c}} \sqrt{\frac{V_{0}}{\beta_{e} M_{t}}}$.

Often there are: $k / k_{h} \ll 1, k_{c e} \sqrt{M_{t} k} / A_{c}^{2} \ll 1$. And the working state that most frequently occurring is zero. $k_{c 0}=0$, so there is $Q_{0}(s)=k_{q 0} x_{v}(s)$. The Eq2 can be written as the Eq3.

$$
x_{p}(s)=\frac{\frac{A_{c}}{k_{c e} k} Q_{L}(s)-\frac{1}{k}\left(1+\frac{s}{\omega_{1}}\right) F_{L}(s)}{\left(\frac{s}{\omega_{r}}+1\right)\left(\frac{s^{2}}{\omega_{h}^{2}}+\frac{2 \xi_{h}}{\omega_{h}} s+1\right)}
$$

Where, $\omega_{1}$ is the turning frequency of a first order differential link, $\omega_{1}=\beta_{e} k_{c e} / V_{0} . \omega_{r}$ is the turning frequency of first order inertial link, $\omega_{r}=k_{c e} k / A_{c}^{2}$.

Displacement sensor transfer function is generally regarded as a pure proportional link. $k_{f}=\frac{u(s)}{x_{p}(s)}$, where, $k_{f}$ is displacement sensor gain. 


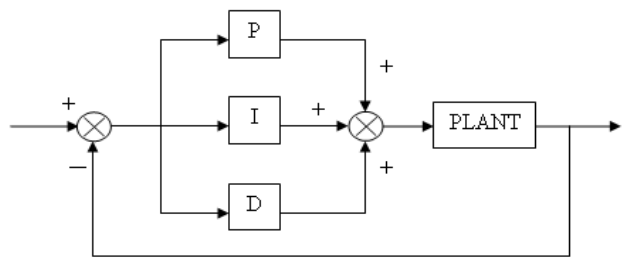

Fig.3. Control diagram of PID

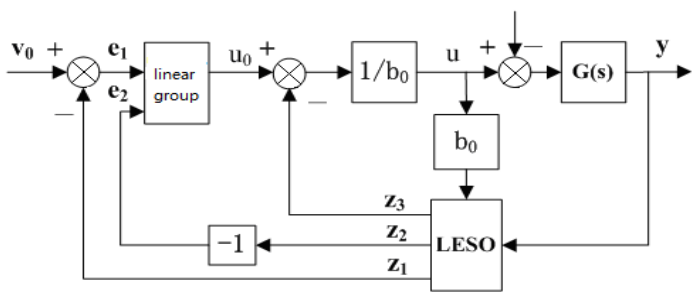

Fig.4. Structure diagram of LADRC

\section{Controller Design}

PID Controller. PID is an effective and simple control algorithm based on information estimation about the deviation of past, present and future. Proportional, integral and differential are linear grouped to constitute controlled variable for controlling controlled process. Its control diagram is shown in Fig.3.

Design of LADRC. For two order nonlinear controlled object:

$$
\left\{\begin{array}{l}
\dot{x}_{1}=x_{2} \\
\dot{x}_{2}=f\left(x_{1}, x_{2}, t\right)+b u \\
y=x_{1}
\end{array}\right.
$$

When $f\left(x_{1}, x_{2}, t\right)$ is unknown, the $f\left(x_{1}, x_{2}, t\right)$ is used as an unknown disturbance, and it is regarded as an unknown expended state variable. That is, make: $x_{3}=f\left(x_{1}, x_{2}, t\right), \dot{x}_{3}=g\left(x_{1}, x_{2}, t\right)$. The $g\left(x_{1}, x_{2}, t\right)$ is also unknown function, then the system becomes a linear system.

$$
\left\{\begin{array}{l}
\dot{x}_{1}=x_{2} \\
\dot{x}_{2}=x_{3}+\mathrm{bu} \\
\dot{x}_{3}=g\left(x_{1}, x_{2}, t\right) \\
y=x_{1}
\end{array}\right.
$$

Establish state observer for this linear system:

$$
\left\{\begin{array}{l}
\varepsilon=z_{1}-y \\
\dot{z}_{1}=z_{2}-\beta_{01} \varepsilon \\
\dot{z}_{2}=z_{3}-\beta_{02} \varepsilon+b u(t) \\
\dot{z}_{3}=-\beta_{03} \varepsilon
\end{array}\right.
$$

Linear extended state observer(LESO) and linear state error feedback control(LSEF) are used to realize the auto disturbance rejection controller, which is called the linear auto disturbance rejection controller. Structure diagram of LADRC is shown as Fig.4.

Where the LESO is used to estimate the object state and the uncertainty perturbation, and LSEF uses the nonlinear or linear configuration of state error feedback and the compensation of the disturbance estimation to generate the control quantity. The equation of LESO is shown in Eq6.The inputs of LESO are $y(t)$ and $u(t)$, and the three system states are $z_{1}(t), z_{2}(t)$ and $z_{3}(t)$.

The composition of the LESF control law is similar to that of PID. Their difference is that the original integral term is replaced by a real time compensation term for the disturbance, this only eliminate static error, and avoid the negative effect of the integral negative feedback. LESF is shown in Eq7. 


$$
\left\{\begin{array}{l}
e_{1}=v_{0}-z_{1} \\
e_{2}=-z_{2} \\
u_{0}=k_{p} e_{1}+k_{d} e_{2}
\end{array}\right.
$$

Design of Fuzzy LADRC. Structure diagram of LADRC based on fuzzy adaptive algorithm is shown as Fig.5. $e_{2}$ and $e_{2}$ are input variables and the output control is nonlinear feedback parameter $\left\{\gamma_{1}, \gamma_{2}\right\}$. These two parameters are similar to the two parameters of the PD controller. $\gamma_{1}$ is proportional coefficient and $\gamma_{2}$ is differential gain.

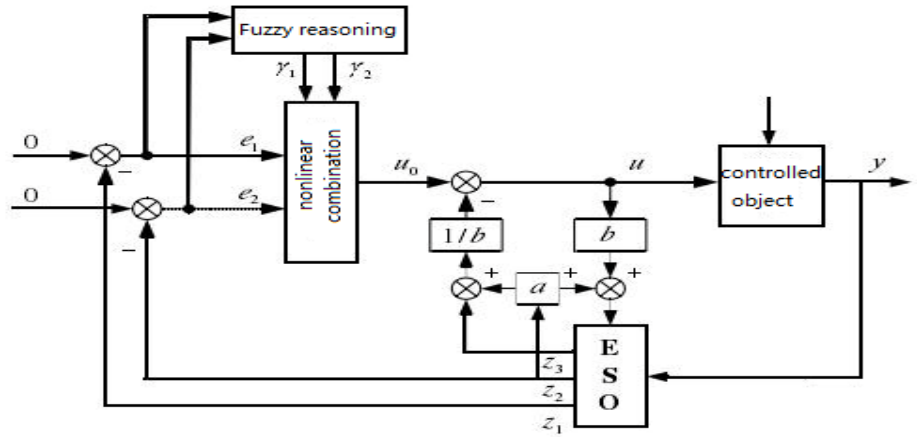

Fig.5. Structure diagram of fuzzy LADRC

\section{Simulation Analysis}

Step response experiments. The experimental and simulated results of step response as shown in Fig.6. Simulation results show that the hydraulic APC system with the auto disturbance rejection controller is better than the traditional PID controller system, and the accommodation time of the LADRC controller with parameter optimization is shorter and the overshoot is smaller, so it has better dynamic characteristics.

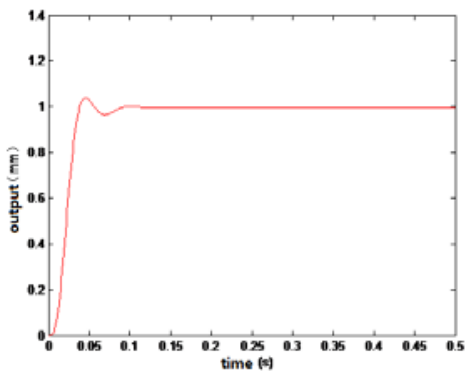

(a)PID controller

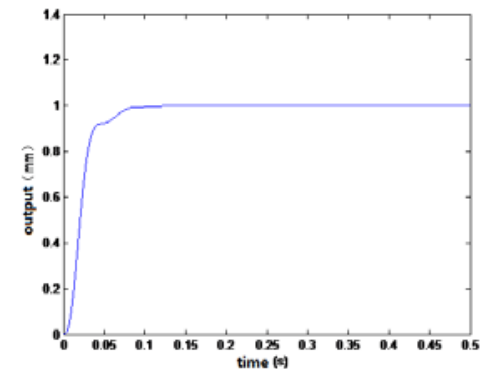

(b)LADRC

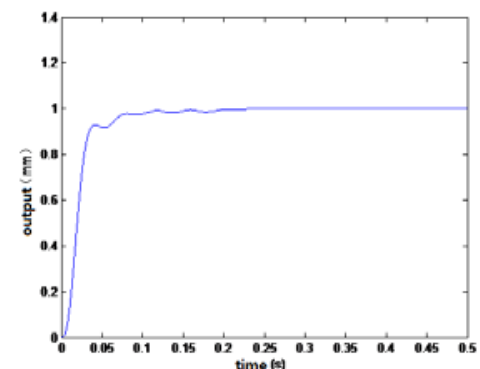

(c)Fuzzy LADRC

Fig.6. The simulated results of step response

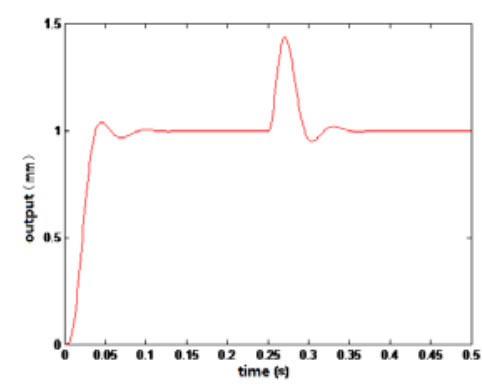

(a)PID controller

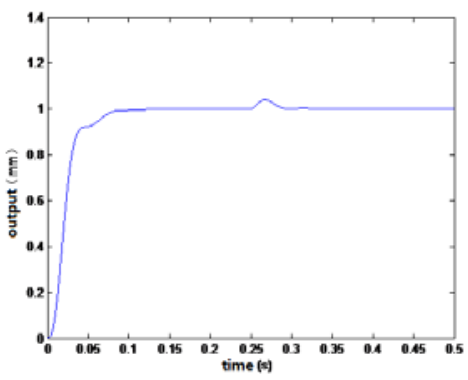

(b)LADRC

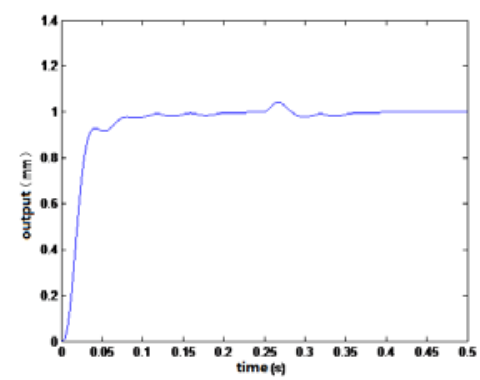

(c)Fuzzy LADRC

Fig.7. The simulated results of step response with load disturbance

Step response experiments with load disturbance. Adding load disturbance to system at the time of $0.25 \mathrm{~s}$ and the simulated results as shown in Fig.7. The impact effect of the HAPC system with the auto disturbance rejection controller is obviously weaker than that of PID control system, 
so this system has better anti-load disturbance capability. Which, the linear auto disturbance rejection HAPC system based on the fuzzy adaptive algorithm parameters optimization, the time required to return to the state after the load disturbance is the shortest, and the ability of anti-load disturbance is the most superior.

Robust experiments. Monte Carlo experiment, also known as the random sampling technique, is an important tool to analyze the robustness of the system. The time domain index is plotted in the overshoot and adjustment time plane, the distance between the region and the origin of the whole shows that the performance index of control system is good or bad. The size of the area reflects the dispersive degree of the performance index of the control system under the change of object parameters. The more the performance index point close to the lower left corner the better the dynamic performance and the more the distribution is intensive the better the time domain performance robustness. Carlo Monte method is used to compare the robustness of HAPC system under deferent control algorithm. The simulated results as shown in Fig.8.

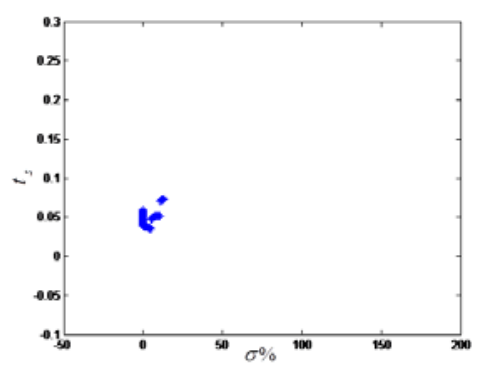

(a)PID controller

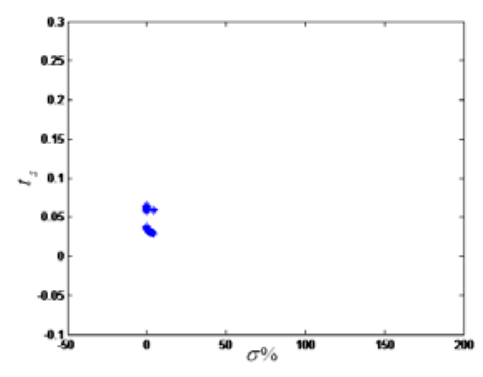

(b)LADRC

Fig.8. The experimental results

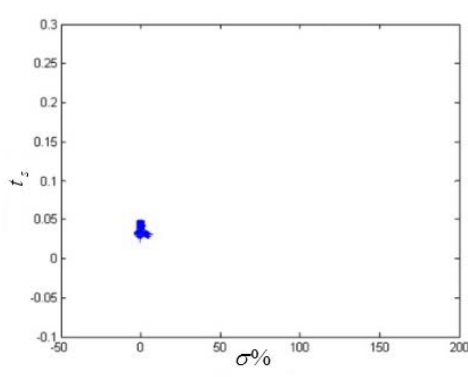

(c)Fuzzy LADRC

As can be seen from Fig.8, the performance index of fuzzy LADRC is the most concentrated and the LADRC second. In general, the robustness of the auto disturbance rejection controller is better than that of the conventional PID controller, and the robustness of the linear auto disturbance rejection controller based on the fuzzy adaptive parameter optimization is the best.

\section{Conclusion}

In this paper, a simulation experiment is carried out based on the three order reduced order model of the hydraulic APC system. The following conclusions are obtained.

(1) The simulation experiment proves that the algorithm of ADRC can effectively overcome the nonlinear and time-varying control problem of the hydraulic APC system. It can also enhance the adaptability of the hydraulic APC system and strengthen the robustness of the system.

(2) LADRC inherits the characteristics of strong anti-interference ability and robustness of the classic auto disturbance rejection controller, while LADRC is more prominent in the fast response of hydraulic APC system, and the structure is more simple and practical.

(3) The fuzzy auto disturbance rejection algorithm is introduced to optimize the parameters of LADRC controller. The results show that the fuzzy algorithm can be used to adjust the parameters of the controller, and simplify the complex process of the parameters setting. It also overcomes the shortcomings that over reliance on experience and knowledge, and the method is simple and practical.

\section{Acknowledgement}

Supported by Fundamental Research Funds for the Central Universities 06105053.

\section{References}

[1] Jingqing Han. From PID technology to auto disturbance rejection control technology [J]. Control Engineering, 2002, 9(3):13-18. 
[2] Xiaofeng Wang. Control of Rough width and flying shear position based on linear ADRC algorithm [D]. Beijing, University of Science and Technology of Beijing, 2007.

[3] Gao Z. Active disturbance rejection control: A paradigm shift in feedback control system design[C]. Minneapolis, MN, United States: IEEE, 2006. 2399-2405.

[4] Hong Chen, Yanbin Gao and Hua Sun. The research and design of adaptive fuzzy auto disturbance rejection controller [J]. Micro computer information, 2008, 24(8):32-33.

[5] Ming Ma. Research on fuzzy control of hydraulic servo control system [D]. Taiyuan, University of Science and Technology of Taiyuan, 2009.

[6] Huaguang Zhang and Xiangping Meng. Basic theory and application of intelligent control [M]. Mechanical Engineering Press,2005. 